\title{
Primeira infância sem açúcar: um direito a ser conquistado
}

\author{
Early childhood without sugar: a right to be ensured
}

La primera infancia sin azúcar: un derecho que hay que conquistar

Juliana Mara Gomes de Assis Nogueira ${ }^{1}$

Ana Maria Costa ${ }^{2}$

Erica Correia Coelho ${ }^{3}$

\begin{abstract}
Resumo
Objetivo: promover a reflexão acerca do impacto do consumo de açúcar na primeira infância à luz da garantia de direitos. Metodologia: o trabalho foi realizado a partir de revisão integrativa de literatura na base de dados PubMed com o uso de descritores específicos. Foram selecionados textos completos em inglês, disponíveis online de 2014 a 2019. Também foram utilizados, de forma complementar, documentos oficiais sobre o perfil nutricional da população e o desenvolvimento das doenças crônicas não transmissíveis em crianças. Resultados: a busca no banco de dados PubMed registrou 36 publicações, das quais onze foram selecionadas para a presente revisão. A fim de relacionar o tema do consumo de açúcar ao direito à saúde da criança, foram consultados também nove documentos jurídicos, onze publicações oficiais de órgãos governamentais, além de livros técnicos, o que possibilitou fundamentar a discussão sobre o impacto do açúcar na saúde da criança como um direito a ser conquistado. Conclusão: apesar do moderno aparato de proteção à saúde e à alimentação da primeira infância pautado pela legislação brasileira, persiste o excesso de açúcar livre na alimentação infantil, o que causa impacto negativo comprovado na prevalência de doenças crônicas não transmissíveis. A alimentação sem sacarose pode ser apontada como um direito da primeira infância devido a sua influência na formação de hábitos, na prevenção de doenças e na qualidade de vida a longo prazo.
\end{abstract}

\section{Palavras-chave}

Açúcares. Criança. Paladar. Nutrição do lactente.

\begin{abstract}
Objective: to reflect on sugar consumption's impact on early childhood in children's rights perspective. Methods: it is an integrative literature review. The authors selected full texts in English by searching articles from 2014 to 2019 in the PubMed database with specific descriptors. To complement the study, it was used official documents on the nutritional profile of the population and the development of chronic non-communicable diseases in children. Results: the PubMed database search recorded 36 publications, of which eleven were selected for this review. To relate the topic of sugar consumption to the right to children's health, nine legal documents, eleven official publications from government agencies, as well as technical books were also consulted, which made it possible to base the discussion on the

\footnotetext{
${ }^{1}$ Especialista em odontopediatria; analista judiciária odontóloga, Superior Tribunal de Justiça, Brasília, DF, Brasil. https://orcid.org/0000-0001-8686-5862. E-mail: junogueira@stj.jus.br.

2 Doutora em Ciências da Saúde; analista judiciária odontóloga, Superior Tribunal de Justiça, Brasília, DF, Brasil. https://orcid.org/0000-0003-1700-5812. E-mail: amc@stj.jus.br

${ }^{3}$ Mestre em Ciências da Saúde; assessora, Gabinete do 5ำ Juizado Especial Cível de Brasília, Tribunal de Justiça do Distrito Federal e Territórios, Brasília, DF, Brasil. https://orcid.org/0000-0002-0210-6584. E-mail: erica.coelho@tjdft.jus.br
} 
impact of sugar on children's health as a right to be conquered. Conclusion: despite the modern apparatus for protecting early childhood health and nutrition by Brazilian legislation, there is still an excess of free sugar in children's diet, which has a proven negative impact on the prevalence of chronic non-communicable diseases. Eating without sucrose can be considered as a right of early childhood due to its influence on the formation of habits, in disease prevention, and on long-term quality of life.

\section{Keywords}

Sugars. Child. Taste. Infant nutrition.

\section{Resumen}

Objetivo: promover la reflexión sobre el impacto del consumo de azúcar en la primera infancia a la luz de la garantía de los derechos. Metodología: el trabajo se realizó a partir de una revisión bibliográfica integradora en la base de datos PubMed utilizando descriptores específicos. Se seleccionaron los textos completos en inglés disponibles en línea desde 2014 hasta 2019. También se utilizaron de manera complementaria los documentos oficiales sobre el perfil nutricional de la población y sobre la evolución de las enfermedades crónicas no transmisibles en los niños. Resultados: la búsqueda en la base de datos PubMed registró 36 publicaciones, de las cuales once fueron seleccionadas para este examen. Para relacionar el tema del consumo de azúcar con el derecho a la salud infantil, se consultaron también nueve documentos jurídicos, once publicaciones oficiales de organismos gubernamentales, además de libros técnicos, que permitieron fundamentar la discusión sobre el impacto del azúcar en la salud infantil como un derecho a conquistar. Conclusión: a pesar del moderno aparato de protección de la salud y nutrición de la primera infancia regido por la legislación brasileña, sigue habiendo un exceso de azúcar libre en la dieta de los niños, lo que ha demostrado tener un efecto negativo en la prevalencia de las enfermedades crónicas no transmisibles. La dieta sin sacarosa puede ser señalada como un derecho de la primera infancia por su influencia en la formación de hábitos, en la prevención de enfermedades y en la calidad de vida a largo plazo.

\section{Palabras clave}

Azúcares. Niño. Gusto. Nutrición del lactante.

\section{Introdução}

A saúde, a alimentação e a educação são direitos sociais, segundo o artigo 6ำ da Constituição Federal (1). Ao analisar o conceito de saúde de forma ampliada, não se pode reduzir saúde à ausência de doença. Em verdade, para se alcançar uma vida saudável é essencial que saúde, educação, direito e alimentação sejam discutidos transdisciplinarmente, à luz do princípio da dignidade humana.

O direito à saúde pode ser considerado como um direito inclusivo que abarca não apenas a assistência em saúde apropriada, mas também atenta para determinantes implícitos à saúde, como a alimentação saudável, o abastecimento adequado de alimentos, habitação, o acesso à água potável e o acesso à educação, especialmente à educação relacionada com a saúde (2). 
A visão da saúde integral, democrática e equânime, associada à educação da população, é importante, sobretudo, ao se considerar a população infantil, diante da sua condição vulnerável de pessoas em desenvolvimento. A Constituição Federal, no artigo 227, sinaliza a responsabilidade da família, da sociedade e do Estado na garantia dos direitos da criança e do adolescente com absoluta prioridade (1). Para viabilizar o cumprimento da regra da absoluta prioridade, foi publicado o Estatuto da Criança e do Adolescente (ECA) (3), que reconhece o estágio peculiar de desenvolvimento característico da infância e da adolescência, o que coloca o público infantil em posição de vulnerabilidade e regulamenta a proteção especial e integral que devem receber (4). Além disso, a partir do ano de 2016, a proteção específica para o começo da vida passou a ser prevista por meio da Lei $\mathrm{n} \cong$ 13.257/16, considerada o Marco Legal da Primeira Infância (5), o que garantiu a criação de programas, serviços e iniciativas dedicadas à promoção do desenvolvimento integral de crianças até os seis anos de idade, bem como à redução das desigualdades.

A alimentação saudável representa uma preocupação para a saúde da criança brasileira, apoiada pelo Direito Humano à Alimentação Adequada e assegurado pelo artigo 25 da Declaração Universal dos Direitos Humanos de 1948 (6). Em consonância, uma alteração na Lei de Diretrizes e Bases da Educação Nacional (LDB) (7) instituiu a obrigatoriedade da educação alimentar e nutricional nas escolas de todo o país (8). Resta, contudo, a aplicação de estratégias que favoreçam práticas nutricionais e capacitação de pais, educadores e cuidadores a fim de beneficiar o desenvolvimento da primeira infância.

Um dos grandes problemas que concorre atualmente com a alimentação adequada é o consumo excessivo de sacarose, sobretudo por crianças e adolescentes. A produção e ingestão de açúcar no Brasil possui um contexto histórico e seu uso industrial o revelou como um produto de grande versatilidade e aplicabilidade, exercendo influência econômica, cultural, psicológica e de paladar nos indivíduos (2). Por outro lado, o aumento de seu consumo está relacionado à maior prevalência de doenças crônicas não transmissíveis (DCNT) na infância, sendo ele causa comum de diversas patologias (9). Por esse motivo, é fundamental a reflexão acerca do consumo de açúcar na primeira infância, relacionando-o às normas jurídicas vigentes.

Diante desse cenário, objetivo deste artigo é fomentar a reflexão acerca do impacto do consumo de açúcar na primeira infância à luz da garantia dos direitos da criança como indivíduo vulnerável, pelas normas do ordenamento jurídico pátrio. 


\section{Metodologia}

Trata-se de uma revisão integrativa da literatura em que foram realizados os passos metodológicos a seguir.

O primeiro passo deu-se pela formulação da questão de pesquisa: qual o impacto do consumo de açúcar na primeira infância à luz da garantia de direitos?

Em seguida, foram selecionados os descritores e as bases de dados a serem utilizadas: o PubMed foi a base de dados escolhida para fundamentar a parte central deste artigo. As palavras-chaves selecionadas a partir do DeCS foram as seguintes: sugars AND child AND taste AND infant nutrition. Os artigos identificados pela estratégia de busca inicial foram avaliados independentemente por dois autores, os quais selecionaram os estudos conforme os critérios de inclusão e exclusão que foram definidos com base na pergunta central que norteou a revisão.

Os critérios de inclusão adotados neste estudo foram: população (criança); tipo de alimentação (alimentos com açúcar adicionado); e desfecho (risco ou não de desenvolver doenças crônicas não transmissíveis relacionadas ao consumo de açúcar). Também estavam entre os critérios de inclusão, textos completos em inglês disponíveis online, de 2014 a 2019. A busca foi realizada em junho de 2020. Para a categorização e sumarização das informações, houve a seleção primária dos artigos pela leitura do resumo. Nova seleção foi realizada pela análise completa dos artigos. Os dados dos estudos incluídos foram agrupados em tabela do Microsoft Word contendo autores, ano de publicação, título, tipo de estudo e uma síntese das conclusões.

Ainda sob o prisma metodológico, para fundamentar as peculiaridades do direito infantil no âmbito dos cuidados em saúde, foi abordado o marco regulatório dos direitos da criança, considerando o direito à saúde, à educação e à alimentação. Foram consultados os seguintes documentos: Declaração dos Direitos Humanos, Convenção dos Direitos da Criança, a Constituição Federal, o Estatuto da Criança e do Adolescente, a Lei de Diretrizes e Bases da Educação e o Marco Legal da Primeira Infância. A estratégia de busca dos documentos jurídicos foi manual, com visita aos sites e endereços eletrônicos das respectivas leis. Foram consultadas publicações oficiais do Ministério da Saúde, Instituto Brasileiro de Pesquisa e Estatística e Organização Mundial de Saúde. A inclusão desses documentos se justifica por apresentarem dados oficiais sobre perfil nutricional da população e sobre o desenvolvimento das doenças crônicas não transmissíveis em crianças, dados indispensáveis para sustentar a ideia central e permitir uma análise argumentativa do 
problema identificado. Também foram utilizados livros técnicos para sedimentar a discussão.

\section{Resultados e discussão}

Foi identificado um total de 36 publicações na base de dados Pubmed. A partir da leitura do resumo e dos textos na íntegra, foram selecionados onze artigos $(n=11)$ que se aproximavam do objetivo proposto pela presente pesquisa, a fim de reportar o impacto do consumo de açúcar na primeira infância. Foi realizado o recorte temporal dos últimos cinco anos (2014-2019) para a realização da busca na base de dados. Dentre os artigos selecionados, predominaram trabalhos de revisão de literatura, sendo duas revisões sistemáticas $(n=2)$ e quatro revisões narrativas $(n=4)$. Os demais trabalhos foram do tipo transversal $(n=2)$, um estudo clínico randomizado, uma pesquisa com abordagem qualitativa e uma publicação com recomendações da Academia Americana de Pediatria (Quadro 1).

Quadro 1. Caracterização das publicações selecionadas na base de dados Pubmed segundo artigo, tipo de estudo e resultados ligados ao tema central da presente pesquisa

\begin{tabular}{|c|c|c|c|}
\hline Título & Autor/Ano & $\begin{array}{l}\text { Tipo de } \\
\text { estudo }\end{array}$ & $\begin{array}{c}\text { Síntese do conteúdo pertinente } \\
\text { ao tema }\end{array}$ \\
\hline $\begin{array}{l}\text { Ontogeny of taste } \\
\text { preferences: basic biology } \\
\text { and implications for health }\end{array}$ & $\begin{array}{l}\text { Mennella } \\
\text { JA,2014 }\end{array}$ & $\begin{array}{l}\text { Revisão } \\
\text { narrativa }\end{array}$ & $\begin{array}{l}\text { Crianças naturalmente preferem } \\
\text { sabores doces e salgados e rejeitam } \\
\text { sabores amargos. Assim, sua biologia } \\
\text { básica os torna especialmente } \\
\text { vulneráveis a alimentos ricos em sal e } \\
\text { açúcares refinados. Porém, } \\
\text { experiências sensoriais no início da } \\
\text { vida podem moldar as preferências } \\
\text { alimentares. Mães que consomem } \\
\text { dietas ricas em alimentos saudáveis } \\
\text { podem moldar o paladar de seus } \\
\text { filhos, porque os sabores são } \\
\text { transmitidos da dieta materna para o } \\
\text { líquido amniótico e para o leite } \\
\text { materno. Desta forma, bebês } \\
\text { amamentados aceitam melhor frutas } \\
\text { e vegetais, ao contrário de bebês } \\
\text { alimentados com fórmula. }\end{array}$ \\
\hline $\begin{array}{l}\text { Vegetable and Fruit } \\
\text { Acceptance during Infancy: } \\
\text { Impact of Ontogeny, } \\
\text { Genetics, and Early } \\
\text { Experiences }\end{array}$ & $\begin{array}{l}\text { Mennella JA, } \\
\text { Reiter AR, } \\
\text { Daniels LM, } \\
2016\end{array}$ & $\begin{array}{l}\text { Revisão } \\
\text { narrativa }\end{array}$ & $\begin{array}{l}\text { Muitas doenças crônicas não } \\
\text { transmissíveis se devem em parte por } \\
\text { escolhas alimentares inadequadas. } \\
\text { Os padrões individuais de } \\
\text { preferências alimentares e } \\
\text { comportamentos alimentares surgem }\end{array}$ \\
\hline
\end{tabular}




\begin{tabular}{|c|c|c|c|}
\hline & & & $\begin{array}{l}\text { e diferem dependendo dos alimentos } \\
\text { oferecidos e dos contextos de } \\
\text { alimentação durante os primeiros } 24 \\
\text { meses de idade, momento no qual } \\
\text { muitas crianças concluíram a } \\
\text { transição alimentar e estão } \\
\text { consumindo dietas semelhantes às } \\
\text { de outros membros da família. À } \\
\text { medida que as crianças fazem essa } \\
\text { transição, as primeiras experiências } \\
\text { com alimentos nutritivos e variedade } \\
\text { de sabores podem maximizar a } \\
\text { probabilidade de elas escolherem } \\
\text { uma dieta mais saudável. O artigo } \\
\text { foca nos períodos iniciais da vida } \\
\text { que, por serem mais sensíveis, } \\
\text { podem modular a aceitação } \\
\text { alimentar. }\end{array}$ \\
\hline $\begin{array}{l}\text { Commercial } \\
\text { complementary food } \\
\text { consumption is } \\
\text { prospectively associated } \\
\text { with added sugar intake in } \\
\text { childhood }\end{array}$ & $\begin{array}{l}\text { Foterek K et } \\
\text { al, } 2016\end{array}$ & $\begin{array}{l}\text { Estudo } \\
\text { transversal }\end{array}$ & $\begin{array}{l}\text { O trabalho mostrou que existe } \\
\text { associação entre a preferência } \\
\text { alimentar por alimentos doces em } \\
\text { idade pré-escolar com alimentação } \\
\text { complementar rica em açúcar no } \\
\text { início da vida. Oferecer } \\
\text { complementação alimentar caseira ou } \\
\text { comercial cuidadosamente escolhida } \\
\text { sem adição de açúcar pode ser uma } \\
\text { estratégia para reduzir a ingestão de } \\
\text { açúcar na infância e posteriormente. }\end{array}$ \\
\hline $\begin{array}{l}\text { The Relationship between } \\
\text { Number of Fruits, } \\
\text { Vegetables, and Noncore } \\
\text { Foods Tried at Age } 14 \\
\text { Months and Food } \\
\text { Preferences, Dietary } \\
\text { Intake Patterns, Fussy } \\
\text { Eating Behavior, and } \\
\text { Weight Status at Age } 3.7 \\
\text { Years }\end{array}$ & $\begin{array}{l}\text { Mallan et al, } \\
2016\end{array}$ & $\begin{array}{l}\text { Estudo } \\
\text { clínico } \\
\text { randomizad } \\
\text { o }\end{array}$ & $\begin{array}{l}\text { O estudo investigou se a exposição a } \\
\text { dietas ricas em frutas e vegetais e a } \\
\text { alimentos processados aos } 14 \text { meses } \\
\text { de idade estava relacionada à } \\
\text { preferência alimentar aos } 3.7 \text { anos. } \\
\text { Os resultados mostraram que os } \\
\text { alimentos utilizados na dieta da } \\
\text { criança com } 14 \text { meses } \\
\text { corresponderam a suas preferências } \\
\text { alimentares aos } 3.7 \text { anos. O estudo } \\
\text { confirmou a hipótese de que as } \\
\text { experiências de sabor no início da } \\
\text { vida influenciam na preferência } \\
\text { alimentar posterior. Isso indica que se } \\
\text { deve introduzir frutas e vegetais aos } \\
\text { bebês e restringir alimentos ricos em } \\
\text { açúcar adicionado. }\end{array}$ \\
\hline
\end{tabular}




\begin{tabular}{|c|c|c|c|}
\hline $\begin{array}{l}\text { Savoring Sweet: Sugars in } \\
\text { Infant and Toddler Feeding }\end{array}$ & $\begin{array}{l}\text { Murray RD, } \\
2017\end{array}$ & $\begin{array}{l}\text { Revisão } \\
\text { narrativa }\end{array}$ & $\begin{array}{l}\text { O artigo reporta a relação do sabor } \\
\text { doce com a criança que começa } \\
\text { antes do nascimento e se prolonga } \\
\text { com a introdução do leite materno } \\
\text { que é naturalmente doce. O autor } \\
\text { pondera que a introdução de bebidas } \\
\text { com açúcar adicionado a bebês pode } \\
\text { aumentar o risco de obesidade no } \\
\text { futuro e dificultar a aceitação de } \\
\text { alimentos amargos ou azedos. }\end{array}$ \\
\hline $\begin{array}{l}\text { Early Taste Experiences } \\
\text { and Later Food Choices }\end{array}$ & $\begin{array}{l}\text { De Cosmi V, } \\
\text { Scaglioni S, } \\
\text { Agostoni C, } \\
2017\end{array}$ & $\begin{array}{l}\text { Revisão } \\
\text { narrativa }\end{array}$ & $\begin{array}{l}\text { A alimentação na fase inicial da vida } \\
\text { é considerada um importante fator de } \\
\text { influência para saúde na vida adulta. } \\
\text { As preferências alimentares são } \\
\text { formadas na infância e podem ser } \\
\text { significativas na prevenção da } \\
\text { obesidade. O gosto pelo sabor doce é } \\
\text { inato e existe uma tendência das } \\
\text { crianças a rejeitar sabores amargos. } \\
\text { Contudo, oferecer alimentos com } \\
\text { diversidade de sabores pode } \\
\text { modificar as preferências alimentares } \\
\text { futuras. Experiências alimentares } \\
\text { saudáveis no período pré-natal pode } \\
\text { transmitir sabores pelo líquido } \\
\text { amniótico e facilitar a aceitação de } \\
\text { diferentes alimentos posteriormente. }\end{array}$ \\
\hline $\begin{array}{l}\text { Complementary Feeding: } \\
\text { A Position Paper by the } \\
\text { European Society for } \\
\text { Paediatric } \\
\text { Gastroenterology, } \\
\text { Hepatology, and Nutrition } \\
\text { (ESPGHAN) Committee on } \\
\text { Nutrition }\end{array}$ & $\begin{array}{l}\text { Fewtrell M et } \\
\text { al, } 2017\end{array}$ & $\begin{array}{l}\text { Revisão } \\
\text { sistemática }\end{array}$ & $\begin{array}{l}\text { Crianças têm preferência alimentar } \\
\text { inata por doce ou salgado. Por outro } \\
\text { lado, rejeitam sabor amargo. } \\
\text { Contudo, evidências mostram que } \\
\text { esta predisposição pode ser } \\
\text { modificada pelo oferecimento } \\
\text { repetido de alimentos sem açúcar } \\
\text { adicionado e com variedades de } \\
\text { frutas e vegetais. Os pais têm } \\
\text { importante papel no estabelecimento } \\
\text { desses hábitos alimentares. }\end{array}$ \\
\hline $\begin{array}{l}\text { Fruit Juice in Infants, } \\
\text { Children, and Adolescents: } \\
\text { Current Recommendations }\end{array}$ & $\begin{array}{l}\text { Heyman MB } \\
\text { et al, } 2017\end{array}$ & $\begin{array}{l}\text { Recomenda } \\
\text { ções da } \\
\text { Academia } \\
\text { Americana } \\
\text { de Pediatria }\end{array}$ & $\begin{array}{l}\text { As recomendações tratam do } \\
\text { consumo de sucos de frutas na } \\
\text { infância. Devido ao sabor doce, os } \\
\text { sucos são facilmente aceitos por } \\
\text { crianças. Contudo, existem efeitos } \\
\text { adversos relacionados ao seu } \\
\text { consumo. A alta concentração de } \\
\text { açúcar contribui com o aumento } \\
\text { calórico do alimento e com o risco de } \\
\text { desenvolver cárie. }\end{array}$ \\
\hline
\end{tabular}




\begin{tabular}{|c|c|c|c|}
\hline $\begin{array}{l}\text { Sugar in Infants, Children } \\
\text { and Adolescents: A } \\
\text { Position Paper of the } \\
\text { European Society for } \\
\text { Paediatric } \\
\text { Gastroenterology, } \\
\text { Hepatology and Nutrition } \\
\text { Committee on Nutrition }\end{array}$ & $\begin{array}{l}\text { Fidler Mis et } \\
\text { al, } 2017 .\end{array}$ & $\begin{array}{l}\text { Revisão } \\
\text { sistemática }\end{array}$ & $\begin{array}{l}\text { A preferência pelo sabor doce é inata } \\
\text { e pode ser modificada ou reforçada } \\
\text { por exposição pré e pós-natal. } \\
\text { Alimentos ricos em açúcar adicionado } \\
\text { podem aumentar o risco de doenças } \\
\text { crônicas não transmissíveis tais como } \\
\text { obesidade, hipertensão, cárie, } \\
\text { diabetes, doenças cardiovasculares e } \\
\text { câncer. Existe a necessidade de } \\
\text { reduzir o consumo de açúcar livre } \\
\text { entre crianças e adolescentes. } \\
\text { Estratégias de educação, dentre } \\
\text { outras, devem ser intensificadas para } \\
\text { atingir esse objetivo. }\end{array}$ \\
\hline $\begin{array}{l}\text { Evidence for high sugar } \\
\text { content of baby foods in } \\
\text { South Africa }\end{array}$ & $\begin{array}{l}\text { Marais NC, } \\
\text { Christofides } \\
\text { NJ, Erzse A, } \\
\text { Hofman KJ, } \\
2019\end{array}$ & $\begin{array}{l}\text { Estudo } \\
\text { transversal }\end{array}$ & $\begin{array}{l}\text { O mercado de alimentos para bebês } \\
\text { da África do Sul é caracterizado por } \\
\text { produtos com alto teor de açúcar, } \\
\text { promovendo um ambiente que } \\
\text { incentiva o desenvolvimento de } \\
\text { preferências de sabor doce e, a longo } \\
\text { prazo, contribuindo para o aumento } \\
\text { da carga de doenças crônicas não } \\
\text { transmissíveis. }\end{array}$ \\
\hline $\begin{array}{l}\text { Parental and Provider } \\
\text { Perceptions of Sugar- } \\
\text { Sweetened Beverage } \\
\text { Interventions in the First } \\
1000 \text { Days: A Qualitative } \\
\text { Study }\end{array}$ & $\begin{array}{l}\text { Morel K et al, } \\
2019\end{array}$ & $\begin{array}{l}\text { Estudo com } \\
\text { abordagem } \\
\text { qualitativa }\end{array}$ & $\begin{array}{l}\text { O trabalho examinou percepções } \\
\text { sobre o consumo de bebidas com } \\
\text { açúcar de adição e estratégias de } \\
\text { intervenção para evitar bebidas com } \\
\text { açúcar adicionado nos primeiros mil } \\
\text { dias de vida. Mensagens com foco } \\
\text { nas consequências para a saúde } \\
\text { infantil e no empoderamento dos pais } \\
\text { para avaliar e selecionar bebidas } \\
\text { mais saudáveis, com base no teor de } \\
\text { açúcar, devem ser testadas em } \\
\text { intervenções, a fim de reduzir o } \\
\text { consumo de bebidas com açúcar de } \\
\text { adição nos primeiros mil dias de vida. }\end{array}$ \\
\hline
\end{tabular}

Fonte: elaborado pelas autoras.

Com a finalidade de relacionar o tema do consumo de açúcar ao direito à saúde, foram selecionados documentos jurídicos $(n=9)$, publicações oficiais do Ministério da Saúde, do Instituto Brasileiro de Geografia e Estatística, da Organização Mundial da Saúde e de outras entidades governamentais $(n=11)$, além de três livros técnicos e o Dicionário de Educação Profissional em Saúde (Figura 1). 
Figura 1. Fluxograma de identificação dos artigos e publicações

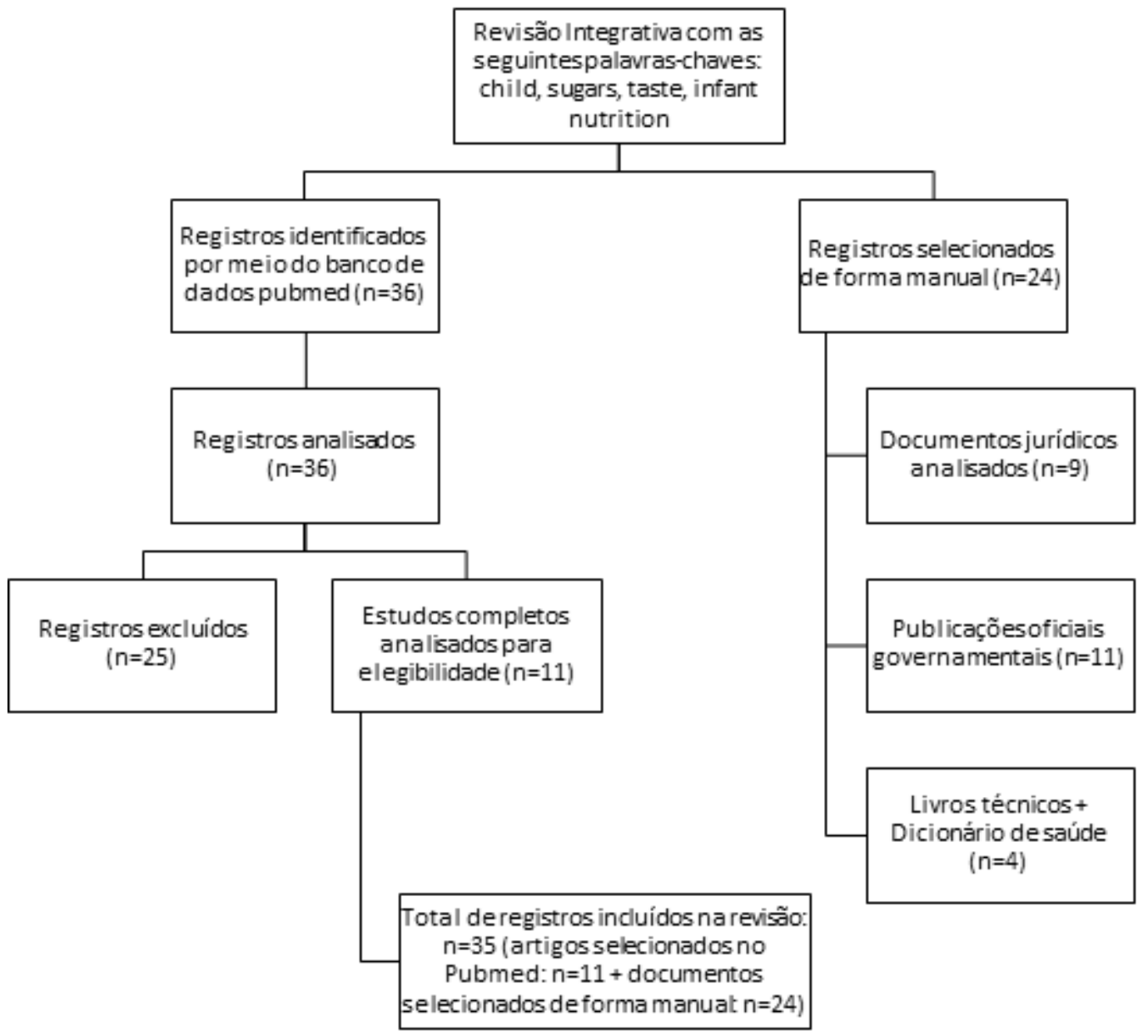

Fonte: adaptado de Costa KB, Silva LM, Ogata, MN (10).

A fase da vida compreendida entre o nascimento e os seis anos de idade, chamada de primeira infância (5), consiste em um período extremamente sensível para o desenvolvimento de diversas habilidades. Trata-se de um período tido como uma janela de oportunidade, porque nele ocorre com maior intensidade o desenvolvimento de aptidões e competências. Nesse momento da vida, há elevada plasticidade cerebral relacionada aos estímulos e às experiências vivenciadas. A primeira infância é uma etapa fundamental na vida do ser humano para que ele possa realizar seu potencial ao longo de sua existência (11).

No que concerne à formação do paladar e do hábito alimentar, sabe-se que é ainda no útero que o feto passa a ser apto a perceber diferentes sabores, pois inicia-se o desenvolvimento dos sistemas sensoriais, dentre eles o olfatório e o gustativo (11). Por essa razão, o consumo pela mãe de alimentos de sabor doce pode ser percebido pelo feto $(11,12)$. Crianças naturalmente preferem sabores doces e rejeitam sabores amargos, o que os torna 
especialmente vulneráveis a alimentos ricos em açúcares $(12,13)$. Já foi demonstrado que sabores e odores presentes no líquido amniótico e no leite materno são oriundos da dieta da mãe e podem moldar a preferência alimentar do bebê $(11,12,14,15)$. Mães que consomem dietas ricas em alimentos saudáveis podem moldar o paladar de seus filhos a aceitarem maior variedade de diferentes sabores ao longo da infância (12). Esses achados reforçam o fato de que as preferências alimentares podem ser moldadas nos primeiros anos de vida da criança $(11,12,14,16)$.

Ao se inferir que hábitos alimentares aprendidos na infância podem interferir nas preferências alimentares por toda a vida, é certo que pais podem desempenhar um papel particularmente importante na formação do paladar de seus filhos (17), oferecendo alimentação complementar sem adição de açúcar, o que é aconselhável não só para a saúde a curto prazo, mas também para manter baixo o limiar de preferência para sabores doces durante toda a vida (12). Destarte, é inegável a influência dos hábitos alimentares sobre a saúde de longo prazo da criança, o que enfatiza a importância do estabelecimento de dieta saudável no início da vida $(11,12,14,18)$.

Embora o sabor doce acarrete uma sensação de prazer durante a alimentação (13), o açúcar adicionado aos alimentos leva ao aumento do valor calórico sem, contudo, acrescentar outros nutrientes essenciais (15). De acordo com recente meta-análise (15), o consumo excessivo de açúcar livre aumenta a chance de DCNT, tais como, obesidade, diabetes, hipertensão, câncer, doença cardiovascular e cárie dentária. Outras pesquisas encontradas na presente revisão confirmam a relação entre o consumo de alimentos adoçados e o risco de desenvolver DCNT, com grande associação entre consumo de açúcar e obesidade $(12,13,19-21)$ e aumento do risco à cárie dentária $(19,21)$, além do efeito do açúcar livre sobre diabetes (19), doenças cardiovasculares $(11,19)$ e câncer (11).

A Organização Mundial de Saúde (OMS) define açúcar livre como aquele adicionado aos alimentos ou bebidas pelo fabricante ou consumidor. Também é considerado como açúcar livre o açúcar extrínseco encontrado no mel, no xarope de milho e nos sucos de fruta concentrados (22). A dieta infantil adequada deve contemplar alimentos que naturalmente apresentam açúcar como parte integrante de sua composição, como, por exemplo, frutas e vegetais $(15,23)$. Segundo a OMS, esses alimentos favorecem o crescimento e o desenvolvimento saudável das crianças. Por outro lado, o uso de alimentos adoçados artificialmente mostrou-se prejudicial à saúde ao longo dos anos $(17,22)$. Além disso, é sabido que o gosto por sabor adocicado é inato $(11,12,13,14)$ e que a oferta de alimentos 
com açúcar livre no início da vida aumenta a chance de preferência alimentar por doce na idade pré-escolar $(19,23)$ e pode interferir com os hábitos alimentares por todo o ciclo de vida $(14,20)$.

Apesar das fortes evidências a respeito dos efeitos adversos do açúcar, o consumo de alimentos doces tem aumentado significativamente nas últimas décadas no Brasil, principalmente entre crianças e adolescentes $(9,24,25)$. A Pesquisa de Orçamentos Familiares reportou que a alimentação do brasileiro se caracteriza pelo baixo consumo de frutas e vegetais e elevado consumo de alimentos calóricos e de bebidas adoçadas, como, por exemplo, sucos artificiais, refrigerantes e refrescos (25). Observa-se também participação crescente de alimentos ultra processados com alto teor de açúcar na dieta brasileira, cujo padrão de consumo varia conforme os grupos etários. Entre os mais novos, é maior o consumo desses alimentos, com tendência à diminuição com o aumento da idade, enquanto o inverso é observado quanto a frutas e hortaliças (24).

O acelerado crescimento do excesso de peso em todas as faixas etárias e de renda deixa clara a necessidade de medidas de controle e prevenção. Entre as crianças, uma em cada três apresentam sobrepeso e 17\% estão obesas (9). Esses dados são preocupantes, já que a criança que é obesa aos quatro ou cinco anos de idade possui maior probabilidade de permanecer com esse problema na fase adulta (2). Estudo transversal mostrou que 70\% dos alimentos industrializados para crianças contêm açúcar livre em sua composição, contribuindo para esse quadro de obesidade e outras DCNT (20).

Diretriz da OMS apontou a cárie dentária como a doença não transmissível mais prevalente do mundo, causando dor, ansiedade, limitações funcionais e consequentemente baixa frequência e mau desempenho escolar entre crianças. O consumo de sacarose está entre os fatores etiológicos principais para o desenvolvimento da cárie (26). A preocupação com a alimentação inadequada da população levou a OMS a sugerir o consumo de açúcar livre a menos de $10 \%$ das calorias totais ingeridas ao dia, sendo preferencialmente menor que 5\% (22). Em consonância, no Brasil o Ministério da Saúde recomenda que não seja utilizado nenhum tipo de açúcar livre no preparo de alimentos nos primeiros dois anos de vida da criança $(27,28)$.

A informação sobre alimentação sem açúcar adicionado é essencial para a saúde da primeira infância, todavia, as recomendações não são seguidas por grande parte da população e o consumo de alimentos adoçados se inicia prematuramente no Brasil. Estudo do Ministério da Saúde revelou que 60,8\% das crianças com idade inferior a dois anos 
consomem biscoitos, bolachas e bolos e que $32,3 \%$ tomam refrigerantes ou suco artificial (29). É importante analisar a oferta inadequada de sacarose na primeira infância sob a perspectiva da privação de direitos, considerando que o consumo excessivo de açúcar aumenta o risco de doenças crônicas não transmissíveis e, desta forma, prejudica o pleno desenvolvimento da criança. São muitos os dispositivos legais que versam sobre o direito da infância, enfatizando que crianças e adolescentes devem ser compreendidos como sujeitos de direitos. A Constituição Federal, em seu artigo 227, considera "dever da família, da sociedade e do Estado assegurar à criança, ao adolescente e ao jovem com absoluta prioridade, o direito à vida, à saúde e à alimentação", entre outros (1).

O conjunto dos princípios específicos que integram o direito da criança e do adolescente no Brasil tem referência, principalmente, na Convenção dos Direitos da Criança, adotada pela Assembleia Geral das Nações Unidas em 1989 e ratificada pelo Brasil em 1990 (30). O documento foi concebido tendo em vista a necessidade de assegurar cuidados especiais à criança, incluindo proteção jurídica adequada, antes e depois do nascimento, em decorrência de sua imaturidade física e mental (31). Ele recomenda a adoção de todas as medidas administrativas, legislativas e de outra índole com vistas à implementação dos direitos da criança.

Ao lado dos princípios e normas instituídos pela Constituição Federal, a Convenção dos Direitos da Criança serviu de fonte de inspiração para a elaboração do Estatuto da Criança e do Adolescente, que entrou em vigor no ano de 1990, o qual também expressa, em seu artigo $3^{\circ}$, a garantia da criança de usufruir de todos os direitos fundamentais inerentes à pessoa humana, com oportunidade de desenvolvimento em condições dignas asseguradas nas dimensões física, emocional, moral e social (3).

Em concordância, no ano de 2016 foi publicado o Marco Legal da Primeira Infância, em cujo artigo $5^{0}$ são elencadas diretrizes para uma série de programas, serviços e iniciativas públicas voltadas à promoção do desenvolvimento integral da primeira infância, com atenção inclusive à saúde, à nutrição e ao consumo consciente (5).

A priorização da primeira infância tem sido discutida em âmbito mundial, conforme mostra o Relatório Final da Comissão para os Determinantes Sociais da Saúde da OMS, do ano de 2010:

A primeira infância proporciona vastas oportunidades para reduzir as desigualdades na saúde, no período de uma geração. A importância do desenvolvimento na primeira infância e da educação para a saúde ao longo do ciclo de vida é um forte imperativo que obriga a uma ação imediata. A 
inércia terá efeitos prejudiciais que podem durar mais que a duração de uma vida (32, p.62).

Em consonância com o excerto acima citado, o documento elaborado para a construção de uma agenda da Primeira Infância da América Latina enfatizou que priorizar a primeira infância é a melhor decisão que um país pode tomar: "é um dos investimentos com maior retorno e permite atingir ao mesmo tempo, objetivos de equidade e eficiência" (33, p.3).

No Brasil, o direito à saúde é viabilizado por meio de políticas públicas de saúde consolidadas pelo Ministério da Saúde. Das políticas gerais de promoção, proteção e recuperação da saúde, a Política Nacional de Alimentação e Nutrição (PNAN), avalia o estímulo a alternativas inovadoras com a superação do modelo tradicional de assistência em saúde pautado pela doença e analisa a necessidade de transposição de desafios, como por exemplo o distanciamento entre os conhecimentos científico e popular (24).

A fim de executar a diretriz de implementação e promoção da alimentação adequada e saudável da PNAN, o Ministério da Saúde elaborou o Guia Alimentar para a População Brasileira, documento que apresenta orientações alimentares oficiais para a população brasileira. O Guia recomenda que seja evitado o consumo de alimentos ultra processados, que possuem ingredientes que os tornam nutricionalmente desbalanceados, com alto teor de açúcar e cujas formas de produção, distribuição, comercialização e consumo afetam de modo desfavorável a cultura, a vida social e o meio ambiente (27).

Em se tratando de primeira infância, importante estratégia educativa contendo recomendações de redução do consumo de açúcar foi lançada pelo Ministério da Saúde no ano de 2019, por meio do Guia Alimentar para Crianças Brasileiras Menores de 2 Anos. Esse guia reforça a fundamentalidade da proteção de crianças e do apoio às famílias para a garantia do Direito Humano à Alimentação Adequada (28).

Outro importante documento de interesse para concretização dos direitos da primeira infância é a Síntese de Evidências para Políticas Públicas do Ministério da Saúde (34), na qual são descritas práticas parentais essenciais para assegurar o desenvolvimento infantil. Esta obra ressalta a relevância de ações voltadas à alimentação e nutrição de crianças na primeira infância e à programas de educação parental. O texto se destaca também por promover a integração entre as áreas de saúde, educação, direito e desenvolvimento social.

Com a publicação da Lei no 13.666, a partir de 2018, a Lei de Diretrizes e Bases da Educação Nacional passou a referir-se à educação nutricional no currículo escolar como 
direito da criança (8). Em se tratando da primeira infância, deve-se levar em conta a condição vulnerável de pessoa em desenvolvimento. Segundo o artigo $8^{\circ}$ da Declaração Universal sobre Bioética e Direitos Humanos (35), indivíduos vulneráveis devem ser protegidos e sua integridade respeitada ao se aplicar conhecimentos científicos relacionados à saúde. A oferta de dieta adequada à primeira infância reflete o respeito pela dignidade humana nas situações em relação às quais a autonomia não é suficiente.

A noção de direitos na visão dos adultos envolve deveres e responsabilidades, porém, na perspectiva da infância, essa mesma noção não pode ser considerada. No caso dela, a garantia de direitos implica o compromisso de atendimento das necessidades fundamentais aos processos de desenvolvimento integral e aprendizagem. Nesse cenário, o Marco legal da Primeira Infância reúne sabiamente um número extenso de necessidades: saúde, alimentação e nutrição, brincar e lazer, proteção contra toda forma de violência e de pressão consumista e prevenção de acidentes (36).

Ao interpretar os Direitos Fundamentais da Criança e do Adolescente, é necessário se colocar na posição deste segmento vulnerável para compreender a gravidade da violação do direito, seja ele de natureza individual, social ou coletiva. A partir disso, pode-se perceber as consequências desse desrespeito, e, desta forma, contribuir em prol da reconstituição do direito violado. De fato, a transgressão ao direito de uma criança é percebida como uma violação ao próprio direito (4).

O direito humano à alimentação adequada está contemplado no artigo $25 \mathrm{da}$ Declaração Universal dos Direitos Humanos de 1948 (6) e é crucial para a fruição de todos os outros direitos, sobretudo o direito à saúde. Ao se entender o processo saúde-doença como o resultado das condições de vida, dentre elas, a alimentação, pode-se apontar estratégias para assegurar a aplicação da legislação. A partir do pensamento de que a saúde não se restringe à ausência de doença, torna-se possível refletir sobre a educação em saúde "como forma do homem reunir e dispor de recursos para intervir e transformar suas condições objetivas, visando a alcançar a saúde como um direito socialmente conquistado" (37, p.155).

A ressignificação de práticas de educação alimentar deve estar focada no acesso à informação científica sobre as causas comuns de patologias, como, por exemplo, o consumo de sacarose, levando-se em consideração a recomendação da PNAN, a qual sugere que os profissionais de saúde devem ser apoiados em seu papel de socialização do conhecimento para viabilizar a decisão de práticas alimentares promotoras da saúde da infância (24). 
É importante considerar que a oferta de sacarose na primeira infância pode ser apontada como afronta à legislação que baliza ações voltadas à promoção do desenvolvimento integral de crianças e adolescentes.

\section{Conclusão}

A saúde como direito fundamental da infância está fortemente consolidada na Constituição Federal, assim como em outros dispositivos específicos do ordenamento jurídico brasileiro, como o Estatuto da Criança e Adolescente e o Marco Legal da Primeira Infância. A alimentação adequada é considerada direito humano de crianças e adolescentes, na medida em que proporciona dignidade à vida e subsídios para o desenvolvimento pleno, garantindo à infância proteção contra doenças potencialmente evitáveis.

Embora estejam garantidos direitos fundamentais à primeira infância por legislação contemporânea e políticas públicas bem elaboradas, existe um quadro grave de prevalência de doenças crônicas não transmissíveis relacionada à alimentação rica em açúcar livre e demonstrada em inquéritos oficiais brasileiros.

A escolha alimentar sem sacarose pode ser apontada como direito da primeira infância e representa um desafio aos serviços e às políticas públicas de saúde já que acarreta forte impacto na vida das futuras gerações, pois pode evitar a formação de hábitos nocivos e o desenvolvimento de doenças, com melhora da qualidade de todo o ciclo de vida do indivíduo.

\section{Referências}

1. Brasil. Constituição da República Federativa do Brasil, 8 de outubro de 1988. Brasília: Senado Federal; 2008.

2. Cruz JI, Carvalho AP, Johns P. O papel da tributação de bebidas açucaradas como forma de garantia do direito à saúde e à alimentação adequada e saudável. In: Corrêa $L$, organizador. Diálogos sobre o direito humano à alimentação adequada [Internet]. Juiz de Fora, MG: Faculdade de Direito da Universidade Federal de Juiz de Fora; 2019 [acesso em: 25.jun.2020]. p. 315-327. Disponível em: https://actbr.org.br/post/dialogos-sobre-odireito-humano-a-alimentacao-adequada/17868/

3. Brasil. Lei no 8.069, de 13 de julho de 1990. Dispõe sobre o Estatuto da Criança e do Adolescente e dá outras providências. Diário Oficial da República Federativa do Brasil [Internet]. Brasília: Presidência da República; 16 jul 1990 [acesso em 25.jun.2020]. Disponível em: http://www.planalto.gov.br/ccivil_03/leis/8069.htm 
4. Dantas TN. Por que sensibilizar o sistema de justiça é tão importante para assegurar os direitos de crianças na primeira infância. In: Henriques I, coordenadora editorial. Primeira infância no sistema de garantia de direitos de crianças e adolescentes: uma experiência a ser replicada. São Paulo: Instituto Alana; 2019. p. 97-108.

5. Brasil. Lei no 13.257, de 8 de março de 2016. Dispõe sobre as políticas públicas para a primeira infância e altera a Lei no 8.069, de 13 de julho de 1990 (Estatuto da Criança e do Adolescente), o Decreto-Lei no 3.689, de 3 de outubro de 1941 (Código de Processo Penal), a Consolidação das Leis do Trabalho (CLT), aprovada pelo Decreto-Lei n 5.452 , de $1^{\circ}$ de maio de 1943, a Lei no 11.770, de 9 de setembro de 2008, e a Lei no 12.662, de 5 de junho de 2012. Diário Oficial da República Federativa do Brasil [Internet]. Brasília: Presidência da República; 8 mar 2016 [acesso em 25.jun.2020]. Disponível em: http://www.planalto.gov.br/ccivil_03/_Ato2015-2018/2016/Lei/L13257.htm

6. Organização das Nações Unidas. Declaração Universal dos Direitos Humanos [Internet]. Paris; 1948 [acesso em 25.jun. 2020]. Disponível em:

https://www.ohchr.org/EN/UDHR/Pages/Language.aspx?LangID=por

7. Brasil. Lei no 9.394 de 20 de dezembro de 1996. Estabelece as diretrizes e bases da educação nacional. Diário Oficial da República Federativa do Brasil [Internet]. Brasília: Presidência da República; 23 dez 1996 [acesso em 25.jun.2020]. Disponível em: http://www.planalto.gov.br/ccivil_03/leis/19394.htm

8. Brasil. Lei no 13.666, de 16 de maio de 2018. Altera a Lei no 9.394, de 20 de dezembro de 1996 (Lei de Diretrizes e Bases da Educação Nacional), para incluir o tema transversal da educação alimentar e nutricional no currículo escolar. Diário Oficial da República Federativa do Brasil [Internet]. Brasília: Presidência da República; 17 maio 2018 [acesso em 25.jun.2020]. Disponível em: http://www.planalto.gov.br/ccivil_03/_Ato20152018/2018/Lei/L13666.htm

9. Brasil. Ministério da Saúde. Agência Nacional de Saúde Suplementar. Vigitel Brasil 2016 Saúde Suplementar: vigilância de fatores de risco e proteção para doenças crônicas por inquérito telefônico [Internet]. Brasília: Ministério da Saúde; 2017 [acesso em 25.jun.2020]. Disponível em: https://www.ans.gov.br/images/Vigitel_Saude_Suplementar.pdf

10. Costa KB, Silva LM, Ogata MN. A judicialização da saúde e o Sistema Único de Saúde: revisão integrativa Cad. Ibero-amer. Dir. Sanit [Internet]. abr./jun., 2020 [acesso em 25.jun.2020] 9(2). Disponível em:

https://www.cadernos.prodisa.fiocruz.br/index.php/cadernos/article/view/635

11. Mennella JA, Reiter AR, Daniels LM. Vegetable and Fruit Acceptance during Infancy: Impact of Ontogeny, Genetics, and Early Experiences. Adv Nutr [Internet]. 2016 Jan 15 [acesso em 25.jun.2020];7(1):211S-219S. Disponível em: https://www.ncbi.nlm.nih.gov/pmc/articles/PMC4717875/

12. Mennella JA. Ontogeny of taste preferences: basic biology and implications for health. Am J Clin Nutr [Internet]. 2014 Mar [acesso em 25.jun.2020];99(3):704S-11S. Disponível em: https://doi.org/10.3945/ajcn.113.067694 
13.Murray RD. Savoring Sweet: Sugars in Infant and Toddler Feeding. Ann Nutr Metab [Internet]. 2017 [acesso em 25.jun.2020];70 (Suppl 3):38-46. Disponível em: https://www.karger.com/Article/FullText/479246\#

14. De Cosmi V, Scaglioni S, Agostoni C. Early Taste Experiences and Later Food Choices.Nutrients [Internet]. 2017 Feb 4 [acesso em 25.jun.2020];9(2):107. Disponível em: https://www.ncbi.nlm.nih.gov/pmc/articles/PMC5331538/

15. Fidler Mis N, Braegger C, Bronsky J, Campoy C, Domellöf M, Embleton ND et al. Sugar in Infants, Children and Adolescents: A Position Paper of the European Society for Paediatric Gastroenterology, Hepatology and Nutrition Committee on Nutrition. Journal of pediatric gastroenterology and nutrition [Internet]. 2017 [acesso em 25.jun.2020]; 65(6):681-696. Disponível em:

https://www.zora.uzh.ch/id/eprint/147718/1/FidlerMisN\%2C_2017.pdf

16. Abanto J, Pinheiro E, Cardoso MA. Dieta e nutrição na fase de primeiros 1000 dias de vida e sua relação com agravos bucais. In: Abanto J, Duarte D, Feres M. Primeiros mil dias do bebê e saúde bucal: o que precisamos aprender! Nova Odessa: Napoleão; 2019. p.1631.

17. Morel K, Nichols K, Nong Y, Charles N, Price S, Taveras E et al. Parental and Provider Perceptions of Sugar-Sweetened Beverage Interventions in the First 1000 Days: A Qualitative Study. Acad Pediatr [Internet]. 2019 Sep-Oct [acesso em 25.jun.2020];19(7):748-755. Disponível em: https://www.ncbi.nlm.nih.gov/pmc/articles/PMC6642844/

18. Fewtrell M, Bronsky J, Campoy C, Domellöf M, Embleton N, Fidler Mis N et al. Complementary Feeding: A Position Paper by the European Society for Paediatric Gastroenterology, Hepatology, and Nutrition (ESPGHAN) Committee on Nutrition. J Pediatr Gastroenterol Nutr [Internet]. 2017 Jan [acesso em 25.jun.2020];64(1):119-132.

19. Foterek K, Buyken AE, Bolzenius K, Hilbig A, Nöthlings U, Alexy U. Commercial complementary food consumption is prospectively associated with added sugar intake in childhood. Br J Nutr [Internet]. 2016 Jun [acesso em 25.jun.2020];115(11):2067-74.

Disponível em: https://pubmed.ncbi.nlm.nih.gov/27079145/

20. Marais NC, Christofides NJ, Erzse A, Hofman KJ. Evidence for high sugar content of baby foods in South Africa. S Afr Med J [Internet]. 2019 Apr 29 [acesso em 25.jun.2020];109(5):328-332. Disponível em:

https://www.researchgate.net/publication/332828228_Evidence_for_high_sugar_content_of _baby_foods_in_South_Africa

21. Heyman MB, Abrams SA. Fruit Juice in Infants, Children, and Adolescents: Current Recommendations._Pediatrics [Internet]. 2017 Jun [acesso em 25.jun.2020];139(6):e20170967. doi: 10.1542/peds.2017-0967. Disponível em: https://pediatrics.aappublications.org/content/early/2017/05/18/peds.2017-0967

22. World Health Organization (WHO). Guideline: Sugars intake for adults and children [Internet]. Geneva: World Health Organization; 2015 [acesso em 25.jun.2020]. Disponível 
em:

https://apps.who.int/iris/bitstream/handle/10665/149782/9789241549028_eng.pdf;jsessionid $=6 \mathrm{FCD} 74 \mathrm{~F} 17 \mathrm{E} 496 \mathrm{D} 8 \mathrm{~F} 6 \mathrm{~A} 9 \mathrm{C} 3 \mathrm{C} 790 \mathrm{~A} 84012 \mathrm{~A}$ ? sequence $=1$

23. Mallan KM, Fildes A, Magarey AM, Daniels LA. The Relationship between Number of Fruits, Vegetables, and Noncore Foods Tried at Age 14 Months and Food Preferences, Dietary Intake Patterns, Fussy Eating Behavior, and Weight Status at Age 3.7 Years. J Acad Nutr Diet [Internet]. 2016 Apr [acesso em 25.jun.2020];116(4):630-7. Disponível em: https://pubmed.ncbi.nlm.nih.gov/26198582/

24. Brasil. Ministério da Saúde. Política Nacional de Alimentação e Nutrição [Internet]. Brasília: Ministério da Saúde; 2013 [acesso em 25.jun.2020]. Disponível em: http://bvsms.saude.gov.br/bvs/publicacoes/politica_nacional_alimentacao_nutricao.pdf

25.Brasil. Instituto Brasileiro de Geografia e Estatística (IBGE). Pesquisa de orçamentos familiares 2008-2009: análise do consumo alimentar pessoal no Brasil. Rio de Janeiro: IBGE; 2011.

26.World Health Organization (WHO). Sugars and dental caries. Who technical information note [Internet]. Geneva: World Health Organization; 2017 [acesso em 25.jun.2020]. Disponível em: https://apps.who.int/iris/bitstream/handle/10665/259413/WHO-NMH-NHD17.12-eng.pdf

27. Brasil. Ministério da Saúde. Secretaria de Atenção à Saúde. Departamento de Atenção Básica. Dez passos para uma alimentação saudável: guia alimentar para crianças menores de dois anos: um guia para o profissional de saúde na atenção básica. $2^{\mathfrak{a}}$ ed., $3^{3}$ reimpr. Brasília: Ministério da Saúde; 2016.

28. Brasil. Ministério da Saúde. Secretaria de Atenção Primária à Saúde. Departamento de Promoção da Saúde. Guia alimentar para crianças brasileiras menores de dois anos. Brasília: Ministério da Saúde; 2019.

29. Brasil. Instituto Brasileiro de Geografia e Estatística. Pesquisa Nacional de Saúde 2013: percepção do estado de saúde, estilos de vida e doenças crônicas - Brasil, Grandes Regiões e Unidades da Federação [Internet]. Rio de Janeiro: Instituto Brasileiro de Geografia e Estatística; 2014 [acesso em 25.jan.2020]. Disponível em: ftp://ftp.ibge.gov.br/PNS/2013/pns2013.pdf

30.Brasil. Decreto no 99.710 de 21 de novembro de 1990. Promulga a Convenção sobre os Direitos das Crianças. Diário Oficial da República Federativa do Brasil [Internet]. Brasília; 21 nov 1990 [acesso em 25.jun.2020]. Disponível em: https://www.planalto.gov.br/ccivil_03/decreto/1990-1994/d99710.htm

31. Albernaz Júnior VH, Ferreira PRV. Convenção sobre os direitos da criança. Revista da Procuradoria Geral do Estado de São Paulo [Internet]. 1998 [acesso em: 25.jun.2020]; 11:445-466. Disponível em: http://www.pge.sp.gov.br/centrodeestudos/bibliotecavirtual/direitos/tratado11.htm 
32. Organização Mundial da Saúde. Redução das desigualdades no período de uma geração. Igualdade na saúde através da ação sobre os seus determinantes sociais. Relatório Final da Comissão para os Determinantes Sociais da Saúde [Internet]. Portugal: Organização Mundial da Saúde; 2010 [acesso em 25.jun.2020]. Disponível em:https://www.who.int/eportuguese/publications/Reducao_desigualdades_relatorio2010.p df

33. Aucilino C, Langou GD. Desenvolvimento Integral na Primeira infância: Agenda de ações para a região da América Latina [Internet]. São Paulo; 2015 [acesso em 26.jun.2020]. Disponível em: https://www.todospelaeducacao.org.br/primeirainfancia/

34. Brasil. Ministério da Saúde. Secretaria de Ciência, Tecnologia e Insumos Estratégicos. Departamento de Ciência e Tecnologia. Síntese de evidências para políticas de saúde: promovendo o desenvolvimento na primeira infância. Brasília: Ministério da Saúde/EVIPNet Brasil; 2016.

35.United Nations Educational, Scientific and Cultural Organization. Universal Declaration on Bioethics and Human Rights [Internet]. Genebra: Unesco; 2005 [acesso em: 25.jun.2020]. Disponível em: http://portal.unesco.org/en/ev.phpURL_ID=31058\&URL_DO=DO_TOPIC\&URL_SECTION=201.html

36. Macedo L. Direito das crianças pequenas ao seu desenvolvimento integral. In: Henriques I, coordenadora editorial. Primeira infância no sistema de garantia de direitos de crianças e adolescentes: uma experiência a ser replicada. São Paulo: Instituto Alana; 2019. p. 123-131.

37. Morosini MV, Fonseca AF, Pereira IB. Educação em saúde. In: Pereira IB, Lima JCF, organizadores. Dicionário de educação profissional em saúde [Internet]. Rio de Janeiro: Fundação Oswaldo Cruz; 2009 [acesso em: 25.jun.2020]. p. 155-162. Disponível em: http://www.sites.epsjv.fiocruz.br/dicionario/verbetes/edusau.html

\section{Colaboradores}

Nogueira JMGA e Costa AM contribuíram com a concepção, redação, análise e interpretação dos dados, revisão crítica e aprovação da versão final. Coelho EC contribuiu com a redação do artigo e revisão crítica de seu conteúdo e aprovação da versão final a ser publicada.

Nogueira JMGA, Costa AM, Coelho EC. Primeira infância sem açúcar: um direito a ser conquistado. Cadernos IberoAmericanos de Direito Sanitário. 2020 out./dez.; 9(4): 51-69. 